\title{
A RATING CRITERION FOR FILM RESISTORS
}

\author{
H. T. LAW \\ University of Edinburgh, $\dagger$ Princess Margaret Rose Hospital, Fairmilehead, Edinburgh, Scotland, U.K.
}

(Received February 13, 1980)

\begin{abstract}
After experimental work has been carried out to determine the required constants, the resistor film temperature may be calculated from its dimensions and power dissipation using an expression which takes account of, and makes a reasonably good approximation for, the heat dissipated by lateral conduction. The arithmetic is simple and well suited to rapid processing using a programmed electronic calculator.
\end{abstract}

\section{INTRODUCTION}

The stability of film resistors depends very much on the film temperature. It is, therefore, of essential interest to the circuit designer to have at his disposal a reasonably accurate criterion by which the relationship between the resistor power dissipation and the film temperature may be established. This is, perhaps, especially true of thin-film resistors since these are most often used in circuit applications where a high degree of stability is required.

It has, of course, always been necessary for the designer of an electronic circuit to have information on the rating of the components he uses. This has formerly been a rather imprecise guideline denoting approximately the stress levels above which the life of the component may be expected to be substantially reduced. As far as the permissible power dissipation of resistors is concerned the decision as to how much the component should be "de-rated" (for high stability or long-life applications) or the recommended rating exceeded (for non-critical or limited life applications) devolved upon the designer and depended on his expertise, built up over a sufficient number of previous failures and a hard-won knowledge of the reliability of each manufacturer - not only in component manufacture and quality control but in accuracy of data sheet preparation.

These methods cannot be carried forward into the design of hybrid film circuits. In the first place hybrid

$\dagger$ The experimental work referred to in this paper was carried out at Ferranti Ltd., Edinburgh using nichrome resistor films deposited on Corning 7059 glass substrates by Balzers Aktiengesellschaft für Hochvakuumtechnik und dünne Schichten, Balzers, Liechtenstein. microcircuits are being used increasingly in applications where precise circuit function is needed, stability requirements are being tightened and accurate predictions of life - not to the point of catastrophic failure but to the end of in-specification behaviour are being demanded. Secondly, the designer, in choosing the resistor geometry, is in effect designing not only the circuit but the component. Finally, the economic pressures which lead to the integration of the maximum number of components within a single hybrid circuit package make inacceptable the generous, over-safe de-rating procedure of the past.

\section{BASIS OF RATING CRITERION}

The most accurate means of determining the temperatures of film resistors (and, for that matter, other components) in a hybrid microcircuit is to solve the partial differential equation defining the steady-state heat flow for the boundary conditions imposed by the circuit geometry, substrate size and package dimensions. Since these are known to a high degree of accuracy (once the circuit is designed and the layout completed) such a solution, with the aid of modern computers, is certainly possible. Suitable software packages for obtaining such solutions have been developed by Dean et al. ${ }^{1,2}$. Nevertheless the procedures are time consuming and can be applied only to the completed design. The prospect of re-iterative design process involving, perhaps, major changes in layout between analyses is not encouraging. Thus manufacturers of film circuits have sought to provide "design guidance rules" whereby the designer, at the drawing-board stage, might make reasonable predictions as to the 


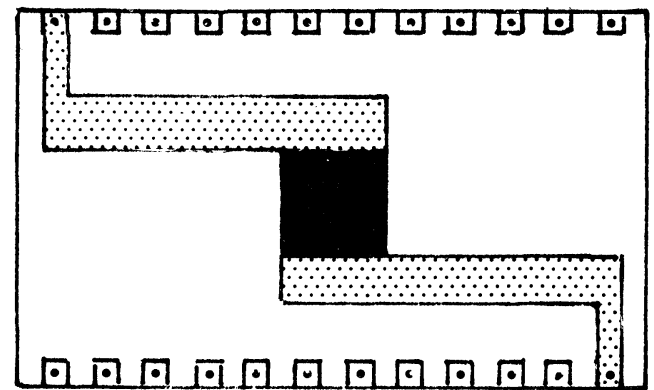

(a) Resistor $4.0 \mathrm{~mm} \times 4.0 \mathrm{~mm}$.

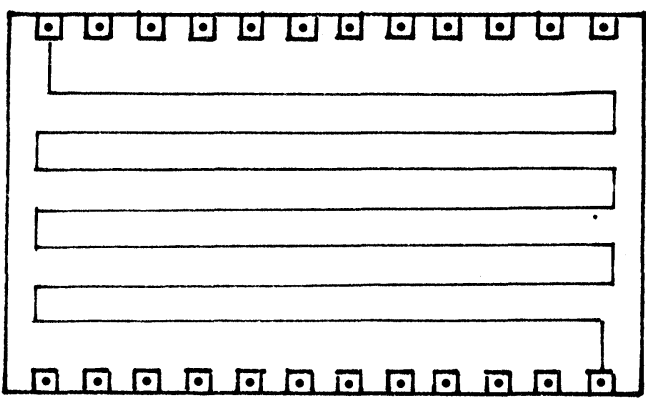

(b) Resistor $160 \mathrm{~mm} \times 0.1 \mathrm{~mm}$.

FIGURE 1 Film resistors where both the film area and the substrate area are the same.

power dissipation of the resistors included in the layout.

These "ratings" are sometimes based on a stated power dissipation per unit area of film. This is clearly prone to serious error; it is obvious that a resistor of given geometry, dissipating a given power, will operate at a lower temperature on a large substrate than on a smaller one. Alternatively, the design rules may base the rating on power dissipation per unit substrate area. Again a hypothetical experiment, comparing two resistors, one almost filling the available substrate area and another only a small part thereof, each of the same dissipation, will show that this basis of prediction may be considerably in error. Finally, if we consider the two examples illustrated in Figure 1, where both the film area and the substrate area are the same, it will be obvious that the resistor shown in Figure 1(b) will, for a given power dissipation, run at a lower temperature than the resistor shown in Figure 1(a). The reason is that the lines of heat flow are by no means normal to planes parallel to the substrate but that substantial laterial diffusion of heat takes place.

The extent to which this lateral heat conduction assists in cooling the film resistor is clearly greater

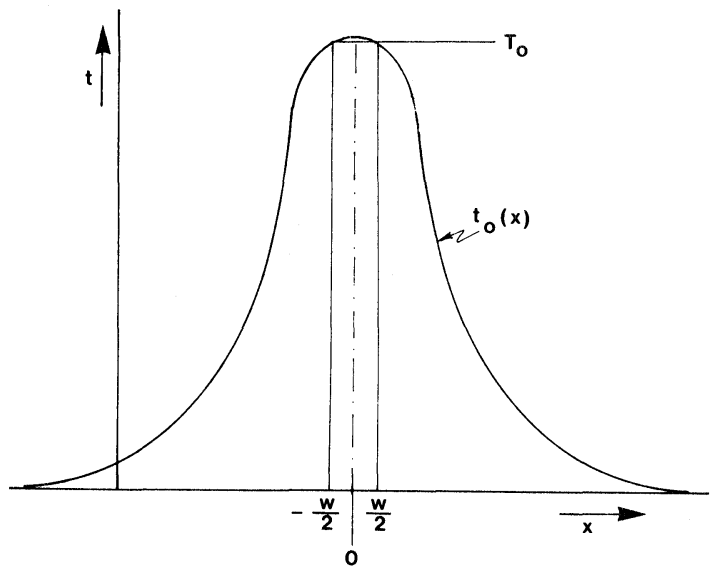

FIGURE 2 Temperature profile at substrate surface for "long" film resistor of width $w$, temperature $T_{0}$.

in the case of long, narrow-width resistors than those of near-square geometry. The total edge-length of the film resistor, as well as its area, therefore seems to be a relevant parameter

The temperature profile at the substrate surface, along a line at right angles to a "long" current carrying resistor is of the form shown in Figure 2. If $x$ is the distance from the midline of the resistor stripe, and $T_{0}$ is the temperature of the resistor, then the temperature is, for this two-dimensional case, a function only of $x$, say $t_{0}(x)$.

It is clear that

$$
t_{0}(x)=t_{0}(-x)
$$

and that, if the heat transfer process is linear (i.e. if radiation can be neglected, which is a valid simplification at the temperatures we are considering) the temperature profile for a different resistor temperature $T$ will be

$$
t(x)=\frac{T}{T_{0}} \cdot t_{0}(x)
$$

If some parallel surface (e.g. the outer surface of the package floor) is maintained at uniform constant temperature $(t=0)$ then the power dissipated, $W$, will be proportional to the area under the curve,

$$
\text { i.e. } W=C \cdot \int_{-\infty}^{+\infty} t(x) \cdot \mathrm{d} x \text { where } C \text { is a constant }
$$

Furthermore, if we assume that the temperature of the resistor film is uniform across its width then

$$
W=C \cdot \int_{-\infty}^{-w / 2} t(x) \cdot \mathrm{d} x+T w+\int_{+w / 2}^{+\infty} t(x) \cdot \mathrm{d} x
$$

where $w$ is the resistor width 


$$
\begin{aligned}
W & =C\left(2 \cdot \int_{w / 2}^{\infty} t(x) \mathrm{d} x+T \cdot w\right) \\
& =C T\left(\frac{2}{T_{0}} \cdot \int_{w / 2}^{\infty} t_{0}(x) \mathrm{d} x\right) \\
& =C T(2 z+w)
\end{aligned}
$$

where $z$ is a constant which can be evaluated if we can determine $t_{0}(x)$, or at least its integral, for any known value of $T_{0}$.

\section{EXPERIMENTAL PROCEDURE}

This was done using an experimental package containing an array of parallel resistors spaced as shown in Table I.

Only the central resistor (R8) was dissipative, the remaining 14 resistors served as thermometers to determine the temperature profile on the surface of the substrate. The temperature coefficient of resistivity of the nichrome film resistors, though small, is finite and stable, and was determined (for each resistor) by heating the entire assembly in a fluorocarbon bath. During the experiment the outer package floor was maintained at a uniform, constant and known temperature by clamping it to a section of copper waveguide through which water is

TABLE I

Disposition of resistors for the determination of surface temperature profile adjacent to single, "long", dissipative resistor

\begin{tabular}{lc}
\hline Resistor No. & $\begin{array}{c}\text { Distance from Midline of Substrate } \\
(\mathrm{mm})\end{array}$ \\
\hline 1 & -2.4 \\
2 & -1.4 \\
3 & -1.0 \\
4 & -0.7 \\
5 & -0.5 \\
6 & -0.3 \\
7 & -0.15 \\
8 & 0 \\
9 & 0.15 \\
10 & 0.3 \\
11 & 0.5 \\
12 & 0.7 \\
13 & 1.0 \\
14 & 1.4 \\
15 & 2.4 \\
\hline
\end{tabular}

All resistors were $0.1 \mathrm{~mm}$ wide, $16.5 \mathrm{~mm}$ long.

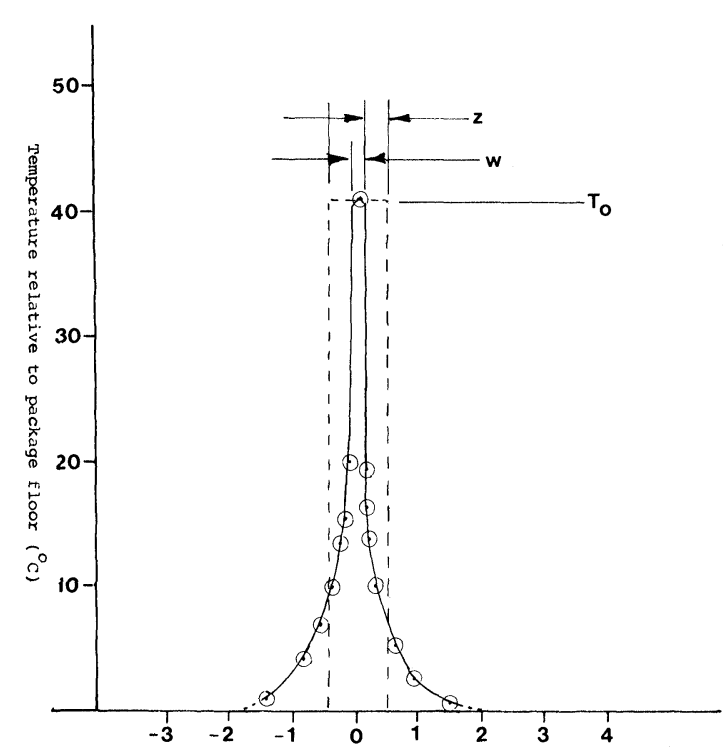

FIGURE 3 Experimental evaluation of $\int t_{0}(x)$.

circulated. Silicone heat-sink compound is used to provide a low thermal impedance at the interface.

The results are shown plotted in Figure 3.

The curve can be integrated by curve-fitting, by graphical methods, or (most simply) by cutting around the curve and weighing the cut-out piece.

For a particular nichrome on glass system the constant $z$ was determined to be $0.69 \mathrm{~mm}$. The physical significance of this constant is illustrated in Figure 3. The rectangle of width $w+2 z$, height $T_{0}$, has the same area as that under the curve $t_{0}(x)$, thus a resistor which is increased in width by $2 z(z$ each side) would dissipate the same power if there were no lateral diffusion of heat. The equivalent dissipative width $(w+2 z)$ allows us to apply simple one-dimensional analysis to the problem.

The constant $\mathrm{C}$ in the above equations was evaluated in a similar procedure, using a substrate pattern consisting of 31 parallel resistors, each $12.7 \mathrm{~mm}$ long, $0.4 \mathrm{~mm}$ wide and evenly spaced at $0.55 \mathrm{~mm}$ pitch. Resistors numbered $1,6,11,16,21$, 26 and 31 were used as thermometers, the remainder were connected in parallel and used as heat dissipating elements. Overall substrate dimensions were $17.8 \mathrm{~mm} \times 17.8 \mathrm{~mm}$. Measurements on the 7 sensing resistors confirmed that the temperature across the medial portion of the substrate was substantially uniform; lines of heat flow in these circumstances are normal to the substrate surface, and the thermal impedance from substrate surface 


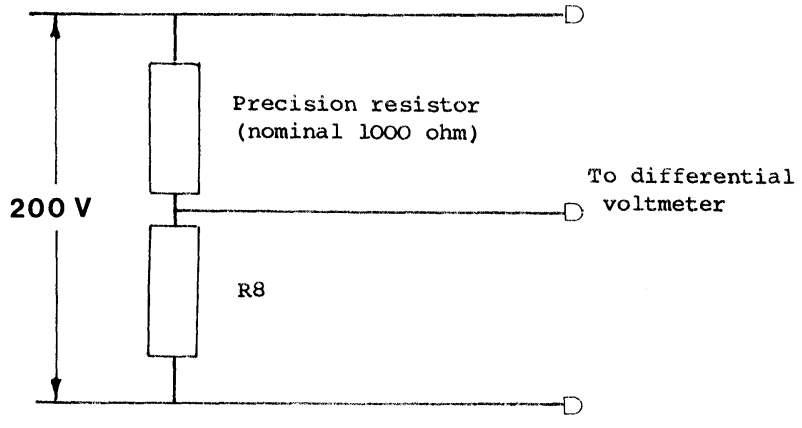

FIGURE 4 Measurement of R8 resistance under load.

to outer package floor determined to be

$14 \mathrm{~K} . \mathrm{cm}^{2} /$ W, i.e. $\mathrm{C}=0.072 \mathrm{~W} \cdot \mathrm{K}^{-1} \cdot \mathrm{cm}^{-2}$.

This result, incidentally, enables a check to be made on the above evaluation of $z$. The central resistor in the array of Table I was $16.5 \mathrm{~mm}$ long $\times 0.10 \mathrm{~mm}$ wide. The temperature could be determined from the measured TCR using a differential voltmeter and the circuit arrangement of Figure 4. The results already presented were obtained with the central (dissipative) resistor at $42^{\circ} \mathrm{C}$ above the outer case temperature, and the dissipated power was $0.712 \mathrm{~W}$, i.e. $0.43 \mathrm{~W} / \mathrm{cm}$ length of resistor.

Recalling the expression for the calculation of power dissipation already obtained

$$
w=C T(w+2 z)
$$

and substituting the values

$$
\begin{aligned}
& C=0.072 \mathrm{~W} \cdot \mathrm{K}^{-1} \cdot \mathrm{cm}^{-2} \\
& T=42^{\circ} \mathrm{C} \\
& W=0.010 \mathrm{~cm} \\
& Z=0.069 \mathrm{~cm}
\end{aligned}
$$

gives

$$
W=0.448 \mathrm{~W}
$$

in very good agreement with the observed result. (The resistor in this example is behaving, in terms of thermal dissipation, as though it were some 15 times wider than its geometric width, if no lateral conduction of heat were taking place.)

\section{APPLICATION}

The discussion so far has dealt with "long" resistor stripes and the method reduces the two-dimensional heat flow problem to a one-dimensional one. For

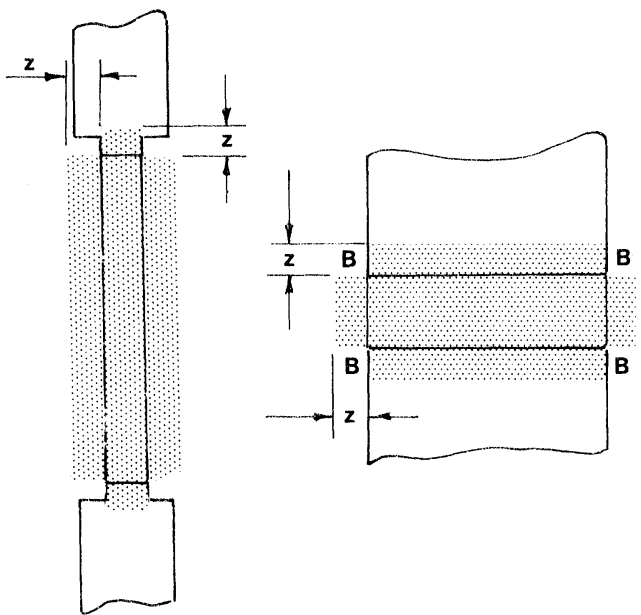

FIGURE 5 Determination of "effective dissipative area" $A_{d}$.

rectangular resistors of moderate aspect ratio an "equivalent dissipative area" $A_{d}$ is derived by the process illustrated in Figure 5. The resistor is considered to be extended a distance $z$ in a direction normal to its boundary along all four sides.

$$
A_{d}=l . w+2 z(l+w)
$$

where $l, w$ are the length and width of the resistor.

This expression takes account of heat flow in directions parallel to the $x$ - and $y$-axes. In practice heat diffusion into the corner areas (" $\mathrm{B}$ " in the figure) will contribute to the effective dissipative area. Neglecting these corners introduces a small error on the "safe" side.

Meander resistors are dealt with in a similar

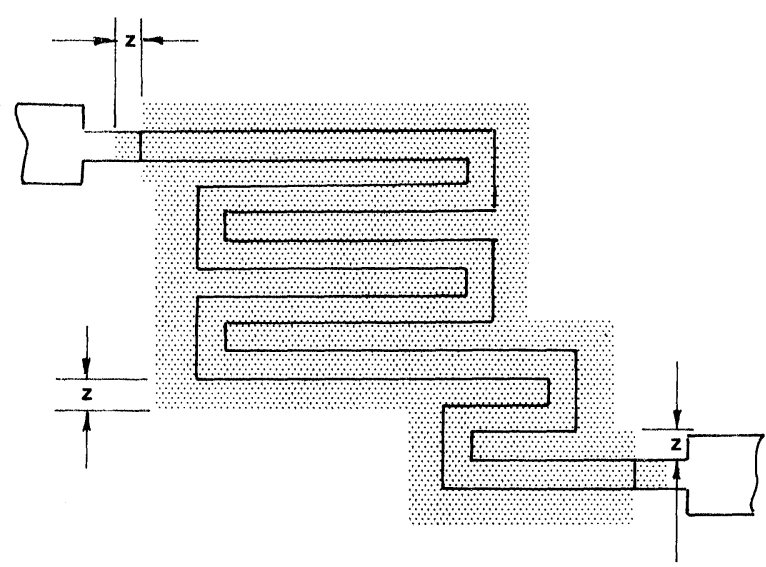

FIGURE 6 Determination of effective dissipative area for meander resistor. 
manner. If the individual "bars" of the meander resistor are closer to each other than a distance $2 z$ the entire resistor may be treated as a single rectangular polygon, and the equivalent dissipative area formed by extension of this polygon by a distance $z$ normal to the boundary as before (see Figure 6).

The necessary arithmetic is well suited to execution on a programmable calculator. If one of the printer cradles now available is used the print-out forms a permanent record, for quality control purposes, that the design has been correctly evaluated and that the resistor temperatures are within the manufacturers declared limits for the performance and stability required.
Since the film resistivity is known it is convenient at the same time to compute the current/unit width to ensure that this falls within previously declared limits, above which electro-migration effects might be expected to begin.

\section{REFERENCES}

1. R. G. Loasby, P. J. Holmes. Handbook of Thick-Film Technology (Electro-chemical Publications Ltd. 1976) Chap. titled "Thermal Design Aspects" by D. J. Dean.

2. P. G. Hambling, D. J. Dean. "Microcircuit Thermal Design Tables and Computer Program for TwoDimensional Layouts". ISHM Conf. on Hybrid Microelectronics, Uni. Tech. Loughborough, Sept. 1975. and IERE Conf. Proc. 31. 

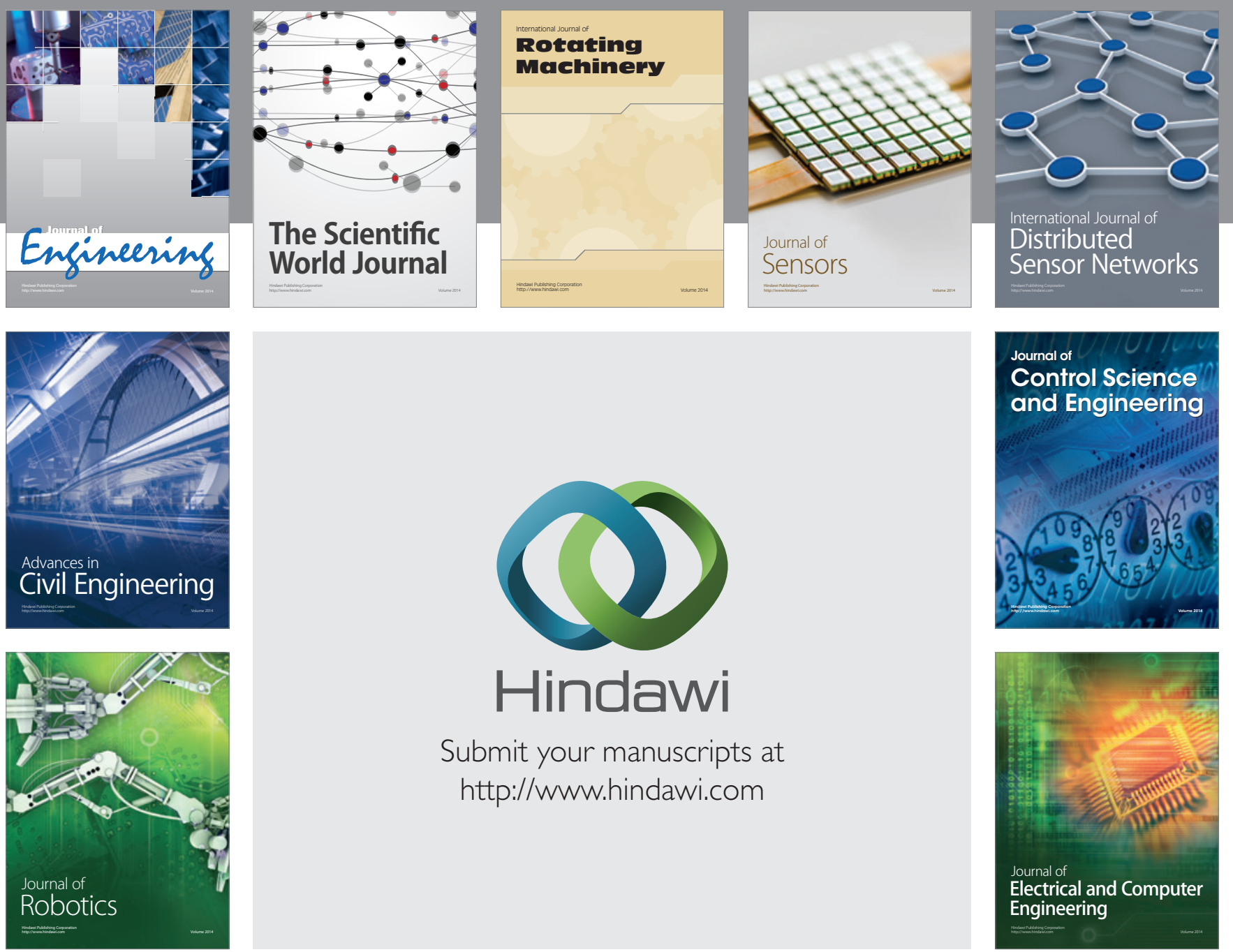

Submit your manuscripts at

http://www.hindawi.com
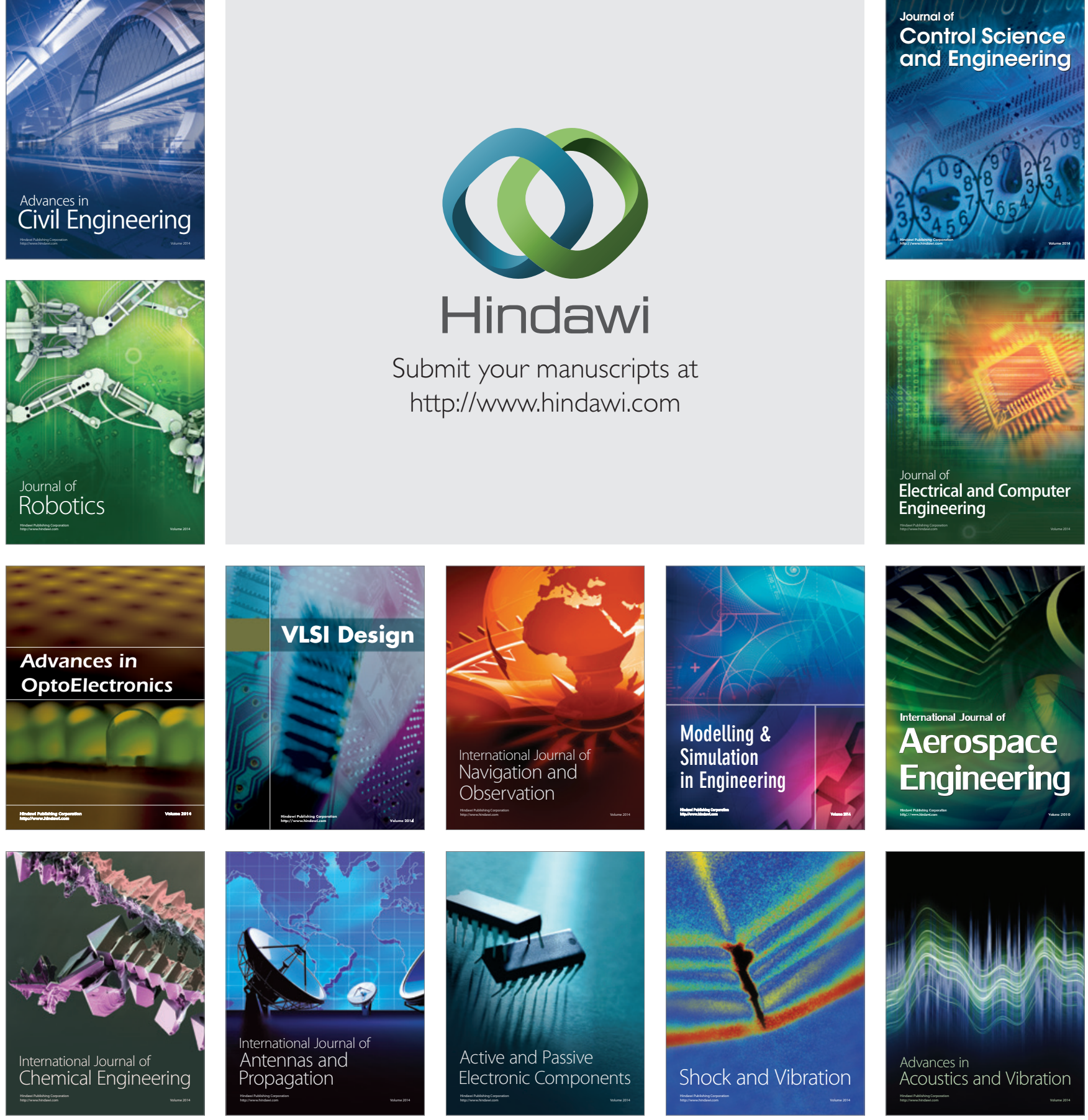